\title{
Structures of a DYW domain shed first light on a unique plant RNA editing regulation principle
}

\author{
Mizuki Takenaka ${ }^{1}$, Sachi Takenaka ${ }^{1}$, Tatjana Barthel ${ }^{2}$, Brody Frink ${ }^{1}$, Sascha Haag $^{3}$, Daniil Verbitskiy ${ }^{3}$, Bastian \\ Oldenkott ${ }^{4}$, Mareike Schallenberg-Rüdinger ${ }^{4}$, Christian Feiler ${ }^{2}$, Manfred S. Weiss ${ }^{2}$, Gottfried J. Palm ${ }^{5}$, Gert Weber ${ }^{2}$ \\ ${ }^{1}$ Department of Botany, Graduate School of Science, Kyoto University, Kyoto, Japan; \\ ${ }^{2}$ Helmholtz-Zentrum-Berlin (HZB), Berlin, Germany; \\ ${ }^{3}$ Molekulare Botanik, Universität Ulm, Germany; \\ ${ }^{4} I Z M B$ - Institut für Zelluläre und Molekulare Botanik, Abt. Molekulare Evolution, University of Bonn, Bonn, Germany; \\ ${ }^{5}$ University of Greifswald, Molecular Structural Biology, Greifswald, Germany \\ gert.weber@helmholtz-berlin.de
}

Pentatricopeptide (PPR) proteins with a C-terminal DYW domain have been characterized as site-specific factors for C to U RNA editing in plant mitochondria and plastids. While substrate recognition is conferred by the repetitive pentatricopeptide (PPR) tract, the exact role of the DYW domain, which can be also recruited to an editing site in trans, has not been clarified. The DYW domain, which was named by the highly conserved last three amino acids, aspartate, tyrosine, and tryptophan, has been proposed as the best candidate to elicit deamination employing a $\mathrm{HxE}(\mathrm{x}) \mathrm{nCxxC}$ zinc ion binding signature. Since $\mathrm{DYW}$ domains share a low sequence conservation with known deaminase structures (from 5 to $19 \%$ residue identities), modelling attempts have been conducted albeit with a limited reliability. Lastly, missing structural information has left the exact function and catalytic properties of DYW domains within the RNA editosome open.

We present structures and functional data of a DYW domain in an inactive ground state and a catalytically activated conformation. DYW domains harbour a cytidine deaminase fold and a C-terminal DYW motif, with catalytic and structural $\mathrm{Zn}$ atoms, respectively. The deaminase fold is interrupted by a conserved domain, which regulates the active site sterically via a large-scale conformational change and mechanistically via the $\mathrm{Zn}$ coordination geometry. Thus, we coined this novel domain 'gating domain' and the accompanying unusual metalloprotein regulation principle of DYW proteins 'gated Zn-shutter'. An autoinhibited ground state and its activation by the presence of either ATP, GTP or the inhibitor tetrahydro uridine is consolidated by differential scanning fluorimetry as well as in vivo and in vitro RNA editing assays. Our observations explain three decades of prior failed attempts to establish an in vitro RNA editing assay and impaired nucleotide binding of DYW domains. In vivo, the framework of an active plant RNA editosome triggers the release of DYW autoinhibition to ensure a controlled and coordinated deamination likely playing a key role in mitochondrial and chloroplast homeostasis.

Keywords: Cytidine Deaminase Mechanism, Metalloprotein Regulation. Plant RNA Editing 